\title{
Proposta de um procedimento operacional padrão para a mitigação das reações transfusionais imediatas em um hospital universitário
}

\author{
Proposal of a standard operating procedure for the mitigation of immediate transfusion reactions \\ in a university hospital
}

Propuesta de procedimiento operativo estándar para la mitigación de reacciones transfusionales

inmediatas en un hospital universitario

Recebido: 10/08/2021 | Revisado: 16/08/2021 | Aceito: 17/08/2021 | Publicado: 22/08/2021

\author{
Aline Bezerra Silva \\ ORCID: https://orcid.org/0000-0003-4628-6844 \\ Universidade Federal de Sergipe, Brasil \\ Empresa Brasileira de Serviços Hospitalares, Brasil \\ E-mail: aline.bs@ebserh.gov.br \\ Adicinéia Aparecida de Oliveira \\ ORCID: https://orcid.org/0000-0002-1551-1992 \\ Universidade Federal de Sergipe, Brasil \\ E-mail: adicineia@ufs.br \\ Juliana de Oliveira Musse Silva \\ ORCID: https://orcid.org/0000-0003-1713-6791 \\ Universidade Federal de Sergipe, Brasil \\ Empresa Brasileira de Serviços Hospitalares, Brasil \\ E-mail: julimusse@ hotmail.com \\ Gleyce Kelly de Brito Brasileiro Santos \\ ORCID: https://orcid.org/0000-0003-4578-5799 \\ Universidade Federal de Sergipe, Brasil \\ E-mail: gkbsantos@hotmail.com \\ Rafael Braga Esteves \\ ORCID: https://orcid.org/0000-0003-4604-6840 \\ Universidade de São Paulo, Brasil \\ E-mail: rafael.braga.esteves@alumni.usp.br \\ Ângela Maria da Silva \\ ORCID: https://orcid.org/0000-0001-9330-537X \\ Universidade Federal de Sergipe, Brasil \\ E-mail: angela.silva910@gmail.com
}

\begin{abstract}
Resumo
Objetivo: Descrever o processo de fundamentação teórica da construção de 2 (dois) Procedimento Operacional Padrão voltado para as medidas de mitigação para as Reações Transfusionais imediatas. Metodologia. Trata-se de um estudo teórico com características qualitativas, descritivas e exploratórias, a qual foi realizada uma imersão na literatura especializada nacional e internacional para subsidiar a produção de um Procedimento Operacional Padrão voltado para as medidas de mitigação das Reações Transfusionais imediatas, desenvolvido entre os meses de março a julho de 2020 realizado em um Hospital Universitário de Sergipe com base nas fragilidades do processo apontadas pela literatura e pela prática laboral. Foi levado em consideração que as RT podem decorrer de três condicionantes: doador, paciente e cuidados prestados. Resultados. O processo de construção do instrumento ocorreu em três momentos: no primeiro, consistiu na revisão da literatura em para localizar evidências científicas qualificadas e atualizadas, sobre o tema estudado; no segundo, foi analisado todo o processo de hemotransfusão do hospital; e no terceiro, foi reunido os registros e/ou anotações acerca das fragilidades processuais e laborais observadas como demandas de profissionais da saúde Considerações Finais: O alcance do cuidado de excelência necessita da ampla divulgação do conhecimento de forma periódica à equipe interdisciplinar, do despertar da necessidade de notificar, da explicitação das fragilidades do processo com os envolvidos para que a comunicação e a identificação do paciente sejam aliadas, do monitoramento da eficácia e das medidas preventivas otimizadas no manejo.
\end{abstract}

Palavras-chave: Enfermagem; Reações transfusionais; Transfusão de sangue; Estudo teórico.

\begin{abstract}
Objective: Describe the theoretical foundation process for the construction of 2 (two) Standard Operating Procedures focused on mitigation measures for immediate Transfusional Reactions. Methodology. This is a theoretical study with qualitative, descriptive and exploratory characteristics, which was immersed in national and international specialized
\end{abstract}


literature to support the production of a Standard Operating Procedure aimed at mitigating measures of immediate Transfusion Reactions, developed between the months from March to July 2020, carried out at a University Hospital in Sergipe, based on the weaknesses of the process pointed out in the literature and in labor practice. It was taken into account that TRs can result from three conditions: donor, patient and care provided. Results. The process of constructing the instrument took place in three stages: in the first, it consisted of a review of the literature in order to find qualified and updated scientific evidence on the studied topic; in the second, the entire blood transfusion process in the hospital was analyzed; and in the third, the records and/or notes about the procedural and labor weaknesses observed as demands of health professionals were gathered. need to notify, explain the weaknesses of the process with those involved so that communication and patient identification are allied, monitoring the effectiveness and preventive measures optimized in the management.

Keywords: Nursing; Transfusion reaction; Blood transfusion; Theoretical studies.

\section{Resumen}

Objetivo: Describir el proceso de fundamentación teórica para la construcción de 2 (dos) Procedimientos Operativos Estándar dirigidos a mitigar las medidas de reacciones transfusionales inmediatas. Metodología. Se trata de un estudio teórico con características cualitativas, descriptivas y exploratorias, que se sumergió en la literatura especializada nacional e internacional para apoyar la producción de un Procedimiento Operativo Estándar dirigido a mitigar medidas de Reacciones Transfusionales inmediatas, desarrollado entre los meses de marzo a julio de 2020, realizado en un Hospital Universitario de Sergipe, a partir de las debilidades del proceso señaladas en la literatura y en la práctica laboral. Se tuvo en cuenta que las TR pueden resultar de tres condiciones: donante, paciente y atención brindada. Resultados. El proceso de construcción del instrumento se llevó a cabo en tres etapas: en la primera, consistió en una revisión de la literatura con el fin de encontrar evidencia científica calificada y actualizada sobre el tema estudiado; en el segundo, se analizó todo el proceso de transfusión de sangre en el hospital; y en el tercero, se recopilaron los registros y / o notas sobre las debilidades procesales y laborales observadas a medida que se recogían las demandas de los profesionales de la salud. Necesidad de notificar, explicar las debilidades del proceso con los involucrados para que la comunicación y la identificación del paciente sean aliadas, monitoreando la eficacia y medidas preventivas optimizadas en la gestión.

Palabras clave: Enfermería; Reacción a la transfusión; Transfusión sanguínea; Estudio teórico.

\section{Introdução}

São inúmeros os pacientes que se beneficiam da transfusão sanguínea, sendo com maior frequência de acordo com a literatura especializada os oncológicos, portadores de afecções hemorrágicas agudas, vítimas de trauma, Coagulação Intravascular Disseminada (CIVD), Púrpura Trombocitopenia Trombótica (PTT), tromocitopênicos e neutropênicos (Cabedal, 2019). Estima-se que paciente internados em Unidade de Terapia Intensiva (UTI) apesentem perda sanguínea de $86,3 \mathrm{ml}$ em quatro dias resultando em anemia dilucional de origem iatrogênica que pode ser percebida com o confronto das variáveis do balanço hídrico e queda de hemoglobina em sete dias de internação (Holland, Peralta, Moss, Feane, \& Uprichard, 2020; Witosz, Wojnarowicz, \& Krzych, 2021).

Pode acontecer também por desordens de hemostasia, distúrbios plaquetários, deficiência de Von Willebrand, dos fatores VIII, XIII e hipofibrinogemia (Raval, Griggs, \& Fleg, 2020). As justificativas para quadros com hemorragia aguda e anemia importantes são explicitadas por (Ajmani, 2020): redução do transporte de oxigênio repercutindo na queda do Débito Cardíaco (DC), dos níveis de hemoglobina (Hb) e da Saturação de oxigênio da hemoglobina (Spo2).

Dessa forma, a transfusão representa $15 \%$ dos procedimentos habituais durante o internamento de pacientes (Panch, Montemayor-Garcia, \& Klein, 2019). É considerado como um procedimento similar a um transplante de órgão sólido, portanto, apesar dos benefícios, o risco a eventos adversos não é inexistente (Chasse et al., 2015). Pode ser comparado como medicamento uma vez que é utilizado com a finalidade terapêutica para o restabelecimento do transporte de oxigênio decorrente de anemia ou da transfusão maciça e da redução dos níveis das proteínas de coagulação e de plaquetas responsáveis pela hemostasia (Kennedy, Harmeníng, \& Justín Rhees, 2019).

Os erros desse procedimento que podem advir das diversas práticas transfusionais conforme a análise de modo e efeito de falha (FMEA) são: informações na solicitação de transfusão, identificação do paciente, da amostra e dos testes de compatibilidade, transfusão de hemocomponentes (HC), preenchimento e envio do documento de controle de transfusão, e 
notificação de reações transfusionais (Mora, Ayala, Bielza, Ataúlfo González, \& Villegas, 2019). Com isso, o processo transfusional exige o fortalecimento da segurança do paciente assim como enaltecimento de aspectos éticos, legais e clínicos (López Lozano, 2015).

Em consonância a esse contexto, o Procedimento Operacional Padrão (POP) busca a padronização do procedimento a partir do detalhamento das atividades desempenhadas pelo executor e do sequenciamento das fases denotando, assim, a qualificação do resultado e redução de falhas (Vilhena et al., 2018). O POP é o recurso que os profissionais de saúde, rotineiramente, buscam para direcionar a prática em hemoterapia e os passos desse recurso devem ser seguidos (Tavares, Barichello, Mattia, \& Barbosa, 2015; Costa, Monteiro, Rodrigues, Monteles, \& Gomes, 2020). Vale ressaltar que o profissional que atua no processo transfusional precisa de proteção jurídica por meio de POP que estandardize responsabilidades e deveres, além disso, este recurso colabora para perseverar a segurança de pacientes no ambiente hospitalar e ambulatorial (Vargas Bermúdez, \& Vargas Bermúdez, 2019). Outro motivo que favorece o avanço da implementação de POP, principalmente nos serviços terciários para a obtenção de diversas selos de acreditações hospitalares ainda que de forma interdependente (Oliveira et al., 2020).

Diante do exposto acerca do processo transfusional e da possibilidade de eventos adversos, este estudo objetivou descrever o método de fundamentação teórica da construção de 2 (dois) Procedimento Operacional Padrão das Reações Transfusionais imediatas voltados para as medidas de mitigação em relação aos cuidados prévios à transfusão e ao ato transfusional.

\section{Metodologia}

Trata-se de um estudo teórico com características qualitativas, descritivas e exploratórias, a qual foi realizada uma imersão na literatura especializada nacional e internacional para subsidiar a produção de um Procedimento Operacional Padrão direcionado para as medidas de mitigação das Reações Transfusionais imediatas, compilando e confrontando os diversos manuais, diretrizes, normas e literatura técnica-científica sobre a temática problematizada (Gil, 2017; Marconi \& Lakatos, 2021).

O presente estudo ocorreu em um Hospital Universitário de Sergipe, local onde as primeiras observações ocorreram e fomentaram a condução desta investigação, o período do estudo compreendeu os meses de março a julho de 2020. O hospital contém 126 leitos e possui uma equipe no setor Agência Transfusional composta por enfermeiros, técnicos de enfermagem, farmacêuticos, biólogos, biomédicos, técnicos de laboratório e hematologistas. A equipe de enfermagem assistencial é a responsável pela instalação, administração, monitoramento e acompanhamento das fases pré transfusional, transfusional e a pós transfusional nas unidades de saúde. No período, o hospital realizava em média 100 transfusões mensais.

O processo de construção do instrumento ocorreu em três momentos: no primeiro, consistiu na revisão da literatura em para localizar evidências científicas qualificadas e atualizadas, sobre o tema estudado; no segundo, foi analisado todo o processo de hemotransfusão do hospital; e no terceiro, foi reunido os registros e/ou anotações acerca das fragilidades processuais e laborais observadas como demandas de profissionais da saúde.

\section{Resultados}

Nesta seção é apresentado os três momentos do processo de construção do Procedimento Operacional Padrão. No primeiro momento foi conduzido uma revisão da literatura para sintetizar as evidências científicas, qualificadas e especializadas quanto as fragilidades, consequências e boas práticas relacionadas ao processo transfusional e a busca eletrônica de POP relacionados a temática em outras instituições (Quadro 1): 
Quadro 1 - Fragilidades do processo Transfusional apontadas pela literatura.

\begin{tabular}{|c|c|}
\hline Fragilidades multifatorial & Consequências \\
\hline $\begin{array}{l}\text { Desconhecimento quanto ao screening de } \\
\text { pacientes para TRALI e TACO (International } \\
\text { Society of Blood Transfusion/International } \\
\text { Haemovigilance Network - ISBT/IHN, 2019). }\end{array}$ & $\begin{array}{l}\text { A incidência é de } 75 \% \text { das reações transfusionais não são comunicadas ao Banco de } \\
\text { Sangue (Gehrie, Hendrickson, \& Tormey, 2015). A incidência de RT permanece } \\
\text { subestimada e as várias razões para isso são: (i) falta de ciência sobre RTs entre os } \\
\text { profissionais, (ii) reações tardias sendo perdidas porque a relação temporal com a } \\
\text { transfusão passa a ser um processo imperceptível, (iii) relato de TACO e TRALI não } \\
\text { modificam o curso do tratamento para pacientes hipoxêmicos, (iv) dificuldade em } \\
\text { identificar RT em pacientes comatosos e (v) sinais e sintomas no paciente podem } \\
\text { sobrepor à condição prévia e gerar fator de confusão (Sahu \& Bajpai, 2020). }\end{array}$ \\
\hline $\begin{array}{l}\text { Identificação incorreta do paciente no formulário } \\
\text { de requisição do HC (Cherem et al., 2018; Polares } \\
\text { et al., 2020). Subestimação da importância do } \\
\text { preenchimento corroborando para RT (Patidar \& } \\
\text { Kaur, 2018). }\end{array}$ & $\begin{array}{l}\text { A ausência do registro das informações dificulta a caracterização das falhas, retarda a } \\
\text { adoção de intervenções terapêutica e reduz a segurança do paciente, que profissional } \\
\text { (Lima, Silva, Rocha, \& Barbosa, 2016). O registro é uma das responsabilidades do } \\
\text { profissional da saúde e da equipe multidisciplinar, que poderia aplicá-lo como evidencias } \\
\text { norteadoras da gestão e da assistência prestada para dimensionar recursos humanos e } \\
\text { físicos de modo a melhorar um ou mais setores, no entanto, mesmo com uma linguagem } \\
\text { pouco técnica, vaga ou incorreta são recorrentes (Bragas, 2015; Negi, Gaur, \& Kaur, } \\
\text { 2015). }\end{array}$ \\
\hline $\begin{array}{l}\text { Erros de transcrição, erro de amostra por não } \\
\text { seguir padrões prévios, ausência de testagem da } \\
\text { tipagem beira leito (Moiz et al.,2020). }\end{array}$ & $\begin{array}{l}\text { Ausência de Tecnologia de Informação para impedir as falhas humanas e a capacidade de } \\
\text { rastrear favorece os eventos adversos (Alotaibi \& Federico, 2017). }\end{array}$ \\
\hline $\begin{array}{l}\text { Ausência da temática na grade curricular, de } \\
\text { capacitações nas instituições de forma permanente } \\
\text { e de protocolos (Carneiro, Barp, \& Coelho, 2017; } \\
\text { Santos, Teixeira, Pereira, \& Silva, 2020; Vieira \& } \\
\text { Santos, 2020). }\end{array}$ & $\begin{array}{l}\text { Falha do processo transfusional e insegurança dos profissionais (Duarte et al., 2017; } \\
\text { Ribeiro et al., 2020). } \\
\text { Carência do processo diante de um profissional que não foi treinado nem qualificado } \\
\text { para atuar na monitorização e no acompanhamento do cliente durante todo o processo } \\
\text { transfusional destoando do preconizado (New Zealand Blood Service, 2016) }\end{array}$ \\
\hline $\begin{array}{l}\text { A falha na verificação dos sinais vitais (Santos, } \\
\text { Santana, \& Oliveira, 2021). As fases essenciais } \\
\text { são: antes da transfusão, dentro de } 15 \text { minutos do } \\
\text { início da transfusão e na conclusão da transfusão } \\
\text { para que haja eficácia no reconhecimento das RT } \\
\text { (Sullivan, Vu, Richardson, Castillo, \& Martinez, } \\
\text { 2015)- sobretudo nos primeiros } 15 \text { min (Gurgel et } \\
\text { al., 2019). Ressalta-se que na fase de pré } \\
\text { administração de HC, os sinais vitais servem de } \\
\text { marcador essencial nos eventos adversos } \\
\text { relacionados à transfusão (Negi et al., 2015). }\end{array}$ & $\begin{array}{l}\text { Inconsistências na monitorização (Reis, Paixão, Perrone, Monteiro, \& Santos, 2016) } \\
\text { diante da ausência da monitorização do paciente nos primeiros } 10 \text { minutos após a } \\
\text { instalação do HC e nos outros segmentos (Santos et al., 2021). As justificativas para o } \\
\text { não cumprimento do registro, da assimilação dos sinais vitais e reconhecimento de uma } \\
\text { RT não podem ser sustentadas pelo trabalho prescrito x real dos enfermeiros nas } \\
\text { instituições nem com alegações de carga de trabalho em demasia ou falha individual, } \\
\text { porém podem ser reduzidas com o engajamento da instituição em garantir capacitações e } \\
\text { elaboração de POP e Protocolos para uniformizar o cuidado prestado e medição de } \\
\text { indicadores (Bezerra, Cardoso, Silva, \& Rodrigues, 2018; Frantz et al., 2020; } \\
\text { Kolankiewicz et al., 2020). }\end{array}$ \\
\hline
\end{tabular}

Nota: A construção do Quadro 1 foi idealizada pelos autores, que se pautaram em uma ampla revisão da literatura especializada na área de hematologia-hemoterapia. Fonte: Autores (2021).

No segundo momento, foi analisar todo o processo de hemotransfusão do hospital cenário da presente investigação, e após observações das dinâmicas dos profissionais da Agência Transfusional (AT) e assistencial nas unidades de internação, foram verificadas as fragilidades da instituição hospitalar, local da pesquisa, onde foi acessada a relação aos processos transfusionais. No terceiro momento, buscou-se a fusão das fragilidades processuais e laborais vivenciadas pela autora e outros profissionais nesse procedimento para a elaboração do POP (Quadro 2):

Quadro 2 - Fragilidades recorrentes do processo transfusional apontados pela prática laboral, 2021.

\begin{tabular}{|l|l|}
\hline Fase pré transfusional & $\begin{array}{l}\text { Quais as informações mínimas para a transfusão; Precisa do termo de consentimento? Histórico do paciente? } \\
\text { Cuidados prévios? Temperatura ideal para o HC no transporte? }\end{array}$ \\
\hline Fase ato transfusional & $\begin{array}{l}\text { Quais parâmetros de sinais vitais para a instalação do HC? Qual é a velocidade de infusão do HC? Como } \\
\text { monitorizar sinais vitais e sinais e sintomas? O que fazer na suspeita de RT? }\end{array}$ \\
\hline Fase pós transfusão & Como comprovar a RT? Como prevenir recorrência de RT? \\
\hline
\end{tabular}

Fonte: Autores (2021). 


\section{Discussão}

A descrição das ações elencadas no instrumento proposto resultou em 2 (dois) POP e três blocos: cuidados gerais para a prevenção da RT, cuidado prévios à transfusão e conduta de mitigação após a constatação da RT. Quanto às atividades executadas e inerentes a cada profissional foram organizadas de forma sequencial desde à solicitação médica do HC até a fase que compreende uma possível RT imediata, 24h após o uso do HC. Logo, o roteiro do POP permite a comunicação efetiva, a padronização sequencial de cada profissional em cada procedimento, reduz os achismos e contribui com o monitoramento de cada ação realizada para garantir resultados qualitativos.

Nos cuidados gerais, destacou-se manter as medidas de higienização das mãos, paramentação e desparamentação mediante o isolamento do paciente, retirada de adornos, assepsia anterior à utilização do acesso venoso e a quaisquer procedimentos. Essas medidas minimizam o risco de transmissão de patógenos e infecção cruzada (Shah Zeb \& Tazeen Saeed Ali, 2020). Outro aspecto que foi realçado no POP é o da orientação do paciente e do acompanhante quanto aos riscos da utilização do HC embasados pela portaria 1820 (Ministério da Saúde, 2009). Outras medidas são identificação do paciente, termo de consentimento para a transfusão.

A identificação do paciente está presente em todas as etapas do processo transfusional. Isso foi reforçado porque o prescritor do $\mathrm{HC}$ não necessariamente será o mesmo que acompanhará o cliente nessa jornada assim como outros profissionais no período de 24 horas podendo contribuir para eventos adversos (Gonçalves, Rocha, Anders, Kusahara, \& Tomazoni, 2016). Portanto, é de vital importância a conferência constante dos identificadores do cliente independente de jornada e da equipe interdisciplinar (Agência Nacional de Vigilância Sanitária - ANVISA, 2016).

Outro cuidado é o termo de consentimento para a transfusão. A equipe interdisciplinar deve atentar- se para o termo de consentimento informado que foi incorporado como fase pré transfusional na instituição por conta dos riscos relevantes a essa temática e que podem comprometer todo o ciclo de segurança do paciente e as taxas de morbimortalidade hospitalar (ANZSBT, 2019; Rodrigues \& Ribeiro, 2021). Com isso, o POP foi embasado nos três pilares da segurança do paciente definido pela Organização Mundial de Saúde - OMS, (2017) que são: a identificação do paciente, a comunicação e a documentação do cuidado. Logo, foi anexado o impresso próprio sobre tais riscos a todos os pacientes/acompanhantes antes da internação e com registro da aceitação ou da recusa desse procedimento (Saito-Benz, Sandle, Jackson, \& Berry, 2019).

Essa instituição não é porta aberta e o internamento dos pacientes com COVID 19 é realizado mediante a regulação do Sistema de Interfederativo de Garantia de Acesso Universal (SIGAU). No contexto da pandemia da COVID 19, isso foi crucial uma vez que o enfermeiro que atua na Unidade de Terapia Intensiva não consegue construir o histórico de enfermagem devido à ausência de acompanhante e os pacientes que são admitidos habitualmente estão sedados.

No que concerne aos cuidados prévios foi considerado relevante a solicitação médica devidamente preenchida, tipo do HC, grupo sanguíneo do paciente, histórico transfusional, sinais e sintomas prévios à transfusão, cuidados específicos para o paciente, HC específico para o diagnóstico do paciente, peso e altura, parâmetros clínicos mínimos para a instalação, sinais e vitais e dos sinais e sintomas prévios à transfusão.

Após a indicação e a solicitação médica do hemocomponente, o enfermeiro deve analisar o formulário de requisição de sangue porque é a garantia da decisão clínica para a transfusão e é definido como requisito de segurança de padrão pela CBS (2017) e ANSM. (2018). As informações contidas não podem conter rasuras ou erros e devem ser legíveis para os dados do paciente, diagnóstico, parâmetros hematológicos atuais, hemocomponente (s) solicitado (s) e histórico de transfusão anterior devendo estar devidamente sinalizadas e com ausência de lacunas que permitam falhas. No POP, existe a orientação que o andamento da solicitação só será concretizado com todos os campos informados de forma legível e sem rasuras (Agência Nacional de Vigilância Sanitária - ANVISA, 2015). 
Outro ponto relevante é considerar as condicionantes para a RT que são: variedades sanguíneas, comorbidades, história transfusional e de alergia medicamentosa (Ministério da Saúde, 2016; Tang, Huang, Lin, \& Yuan, 2020). Assim como pacientes com potencial de aloimunização como os politransfundidos e as multíparas (Moncharmont, 2018; Garraud et al., 2018). Pode ocorrer também por incompatibilidade no sistema ABO e do fator Rh como no caso de plaquetas incompatíveis que podem potencializar em 1,5 a 2 vezes às RT em relação as plaquetas compatíveis, especialmente a incompatibilidade maior podendo ser subestimada (Bastos, 2015; Malvik, Leon, Schlueter, Wu, \& Knudson, 2020; Dunbar, 2020). No caso do plasma, não carece de compatibilidade do fator Rh (Kennedy et al., 2019). O enfermeiro deve analisar todas as variáveis nessa etapa e repassar as informações para outros profissionais de saúde conforme POP.

No POP, o screening do paciente deve ser realizado principalmente pelo médico e pelo enfermeiro. Os fatores predisponentes do paciente podem ocasionar uma RT o que evidencia a capacidade do planejamento das ações anteriormente à transfusão tornando-a prevenível e não deve ser descaracterizada (Aubron, Aries, Le Niger, Sparrow, \& Ozier, 2018). As condições do doente devem ser identificadas, estudadas e registradas (Saito-Benz et al., 2019). Entre elas, denotar as patologias que exigem o hemocomponente adequado (Ministério da Saúde, 2015; Canadian Blood Services - CBS, 2017; Ajmani, 2020,).

Uma vez identificado, por exemplo, risco para Sobrecarga Volêmica Associada à Transfusão (TACO) aspectos como volume de infusão, Pressão Venosa Central (PVC), Débito Urinário (DU), Balanço Hídrico (BH), uso de diurético prévio ou o uso do $\mathrm{HC}$ em períodos de hemodiálise (HD) precisam ser revistos e assimilados pela equipe interdisciplinar previamente ao processo transfusional. A Lesão Pulmonar Relacionada à Transfusão (TRALI) segue a abordagem de condições comórbidas específicas (Roubinian, 2018). Essas condições comórbidas são explicitadas no POP, de acordo com o International Society of Blood Transfusion/International Haemovigilance Network - ISBT/IHN, (2019). Ou ainda em verificação do uso de antihipertensivos prévios como Inibidores da Enzima de Conversão da Angiotensina (IECA) a fim de reduzir o potencial de reação Hipotensiva em pacientes na fase pré operatória (Yoon, Abdullah, Elia, \& Herman, 2018).

Deverá ser checada a condição clínica do paciente prévia à infusão o HC e os parâmetros clínicos mínimos para a autorização da instalação na instituição são: Pressão Arterial (PA) <160/100mmHg, Frequência Respiratória (FR) <30ipm, Pulso (P ou FC) $<130 \mathrm{bpm}$, Temperatura ( $\mathrm{T}$ ) $<37,8^{\circ} \mathrm{C}$. Caso apresente alguma incompatibilidade, o médico de plantão (MP) ou o médico assistente (MA) deverá avaliar e autorizar à administração do HC. Só instalar o HC, mediante a compatibilidade dos sinais vitais do paciente aos parâmetros mínimos adotados pela instituição (Empresa Brasileira de Serviços Hospitalares EBSERH, 2019).

Após essa etapa, deve-se providenciar o acesso calibroso e via exclusiva uma vez que pacientes críticos utilizam diversos medicamentos incompatíveis e isso reduz a possibilidade de ocorrer a Hemólise não Imune (HNI). A HNI é uma reação evitável, pois retrata o rompimento da membrana eritrocitária durante ou após a transfusão ocasionando a liberação da hemoglobina livre no plasma do receptor gerando hemoglobinemia e hemoglobinúria (Veiga \& Rojas, 2018). A hemólise decorre da sensibilidade dos produtos sanguíneos a lesões mecânicas, térmicas e osmóticas e são derivadas de desconhecimento técnico (imperícia) como o uso de bombas de infusão incompatíveis com a prática transfusional, pressurização à bolsa do $\mathrm{HC}$, acessos de fino calibre, uso de medicamentos e soros inconciliáveis com o procedimento. Isso só prova que as instituições precisam garantir capacitações para impedir erros e exterminar esses riscos (Harewood, Ramsey, \& Master, 2021).

$\mathrm{Na}$ fase de recepção do HC, deve-se proceder a conferência das condições ideais da temperatura da caixa térmica e do acondicionamento de acordo com o tipo de HC: Concentrado de Hemácias $(\mathrm{CH})$ e Plasma Fresco descongelado $\left(1-10{ }^{\circ} \mathrm{C}\right)$, Concentrado de Plaquetas (CP), Crioprecipitado descongelado e Concentrado de Granulócitos $\left(22 \pm 2{ }^{\circ} \mathrm{C}\right)$. Ressalta-se que o Crioprecipitado e o Plasma Fresco Congelado após o descongelamento (temperatura $37^{\circ} \mathrm{C}$ ) devem ser usados prontamente a fim de evitar precipitações (Simon, McCullough, Snyder, Solheim, \& Strauss, 2016; Ministério da Saúde, 2016; Castrillo, 
Arroyo, Romón, \& Rivera, 2020). Deve-se respeitar a validade dos HC após liberação para uso: CH-30 minutos, PF descongelado e CG (24 horas), CP se mantido em um agitador de plaquetas por 24 horas e Crioprecipitado (06 horas). Um contraponto é que apenas $12 \%$ dos hospitais controlam adequadamente a temperatura de transporte da agência transfusional à unidade do paciente. Considerar que caso o HC ultrapasse a validade e as condições de temperatura não sejam asseguradas devem ser recolhidos e devolvidos à equipe da agência transfusional a fim de evitar eventos adversos e reduzir a eficácia do HC (Agência Nacional de Vigilância Sanitária - ANVISA, 2015; Castrillo, Arroyo, Romón, \& Rivera, 2020; Kennedy et al., 2019; Dunbar, 2020).

Ao receber o HC, os enfermeiros da unidade do paciente e da Agência Transfusional devem conferir com checagem dupla ou positiva; I nome completo do receptor, sem abreviaturas; II- sexo, idade e peso; III - número do prontuário do paciente e data de nascimento; IV - identificação do serviço de saúde, localização intra-hospitalar e número do leito; Vdiagnóstico e indicação da transfusão; VI - resultados dos testes laboratoriais que justifiquem a indicação do hemocomponente; VII - caráter da transfusão (programada, rotina, urgência, emergência); VIII - hemocomponente solicitado, com o respectivo volume ou quantidade; IX - data, assinatura e número de inscrição no Conselho Regional de Medicina do médico solicitante; e XI - antecedentes transfusionais, gestacionais e reações à transfusão e a medicamentos assim como compatibilidade entre o HC e o tipo sanguíneo do paciente conforme a Portaria nº 158, de 4 de fevereiro de 2016 (Ministério da Saúde, 2016).

Avaliar o HC quanto à coloração, sinais de hemólise, a integridade do sistema, a presença de hemólise ou de coágulo e a data de validade, avaliar o tipo de grupo sanguíneo do paciente e do HC (Ministério da Saúde, 2016). Ao instalar o hemocomponente, assistir o paciente a beira leito no período de $15 \mathrm{~min}$ e com o gotejamento $2 \mathrm{ml} / \mathrm{min}$ do $\mathrm{HC}$ ou ainda na infusão dos primeiros $50 \mathrm{ml}$, e registrar os sinais vitais no prontuário com 1h, $2 \mathrm{~h}, 3 \mathrm{~h}$ e $4 \mathrm{~h}$. Sinalizar alguma intercorrência, tais como: sinais de alteração na pele/mucosa, respiratória, temperatura, cardiológica e renal (Canadian Blood Services - CBS, 2017; Gorski L, Hadaway L, Hagle ME, McGoldrick M, Orr M, 2016).

Após os 15 min e caso o paciente não apresente intercorrência, atentar-se para o tempo de infusão de cada unidade de CH deve ser de 60min a 120 minutos em pacientes adultos e com tempo máximo de 240 min e no caso de plasma, crioprecipitado e plaquetas deverá ser de aproximadamente 30min a $60 \mathrm{~min}$ em pacientes adultos e pediátricos. Em pacientes pediátricos, não exceder a velocidade de infusão de $20-30 \mathrm{ml} / \mathrm{kg} / \mathrm{h}$ (Agência Nacional de Vigilância Sanitária - ANVISA, 2015; Dunbar, 2020). A administração do HC precisa ser cuidadosa com o gotejamento do $\mathrm{HC}(1 \mathrm{ml}=15$ gotas/min), não devendo ultrapassar uma infusão $2 \mathrm{ml} / \mathrm{min}$ ou $2 \mathrm{ml} / \mathrm{kg} / \mathrm{h}$ em pacientes de alto risco e não poderá ser administrado de forma simultânea. Ressalta-se assim a importância da avaliação do peso e da altura do paciente na fase pré transfusão e administração. O controle da infusão pode reduzir o potencial para Hipotermia principalmente em crianças por ser pesodependente (Aubron et al., 2018; Guerado, Medina, Mata, Galvan, \& Bertrand, 2016; Medecins Sans Frontieres - MSF, 2019; Najafpour et al., 2017) e para TACO (Hulse et al., 2020; Semple, Rebetz, \& Kapur, 2019; Spilka \& Goobie, 2020).

$\mathrm{Na}$ etapa de instalação, deve-se atentar-se para o manejo inadequado dos dispositivos associados pelos profissionais, que é outra RT fruto de iatrogenia, a Embolia Aérea. Durante o ato transfusional, os pacientes elegíveis à TACO ou a TRALI ou aqueles que apresentarem dispneia, alteração dos parâmetros ventilatórios ou de suporte ventilatório deverão ser submetidos a gasometria e a um radiografia de tórax na janela de 12h ou 6h após a instalação do $\mathrm{HC}$, respectivamente, facilitando a qualidade do cuidado e a eficácia da transfusão (Roubinian, 2018; Vlaar et al., 2019).

Outro aspecto de forma abrangente, os sinais vitais, os sinais e sintomas, as condições compridas, a condição física inicial do paciente e os exames subsidiarão a confirmação da RT, por isso, é crucial a vigilância visual e técnica (Center for Disease Control/Division of Healthcare Quality Promotion - CDC/DHQP, 2018; Ribeiro et al., 2020; Saito-Benz et al., 2019). A ocorrência dos sinais e sintomas podem caracterizar uma RT embora o reconhecimento possa ser insuficiente e constam em diversos protocolos internacionais e nacional. (Schaffhausser, Faria, Suano-Souza, \& Sarni, 2020). 
Entre os sinais vitais, a taquicardia e a dispneia podem ser relacionados ou serem fatores independentes para pacientes anêmicos na fase pré transfusional (Adkins, Lawicki, Johnson, \& Eichbaum, 2019). Nesse sentido, a taquicardia pode ser observada como superior a $100 \mathrm{bpm}$ e a taquipneia superior a $30 \mathrm{ipm}$ (Kennedy et al., 2019). A taquicardia é induzida pela redução do oxigênio tecidual como mecanismo compensatório da anemia (Whittaker et al., 2019).

A febre deve ser considerada com uma alteração de ao menos com $1^{\circ} \mathrm{C}$ em relação ao valor pré transfusional ou acima de $38^{\circ} \mathrm{C}$ (Agência Nacional de Vigilância Sanitária - ANVISA, 2015; Center for Disease Control/Division of Healthcare Quality Promotion - CDC/DHQP, 2018). A alterações hemodinâmicas compreendem a alteração superior ou inferior a 20 mmHg e $20 \%$ na pressão arterial sistólica (Lim, Kim, \& Park, 2020). Entretanto, foi considerado como aceitáveis as alterações durante $o$ ato transfusional como: $\pm 0,5{ }^{\circ} \mathrm{C}$ para a temperatura, \pm 5 ipm na frequência respiratória, $\pm 10 \mathrm{bpm}$ para a frequência cardíaca e $\pm 20 \mathrm{mmHg}$ para a pressão arterial embora os sinais e sintomas façam parte do contexto e devem ser assimilados de forma conjunta (Knopfelmacher, 2020).

Em termos de reação Hipotensiva (HIPOT) como RT deve-se considerar como critério a idade do paciente. No caso da imputabilidade confirmada, sem outras causas que possam interferir na hipotensão exceto a transfusão, ocorre em menos de 15 min após o início da transfusão que reverte após 10 minutos da interrupção da transfusão e medidas de suporte. Para conceituar, configura-se como reação hipotensiva: acima de 18 anos de idade (queda maior ou igual a $30 \mathrm{mmHg}$ e aferição menor ou igual a $80 \mathrm{mmHg}$ da pressão arterial sistólica); entre 1 a 18 anos de idade (queda maior que 25\% da pressão sistólica basal); em < 1 ano de idade ou com peso corpóreo inferior a $12 \mathrm{~kg}$, queda maior que $25 \%$ do valor basal da pressão arterial sistólica, diastólica ou média. Deve-se eliminar outras etiologias que justifiquem a hipotensão (Agência Nacional de Vigilância Sanitária - ANVISA, 2015; Center for Disease Control/Division of Healthcare Quality Promotion - CDC/DHQP, 2018; Serious Hazards of Transfusion - SHOT, 2019).

Na suspeita de uma RT, interromper a administração do HC e só reinfundir em casos de reação alérgica leve tratada (Instituto Português do Sangue e da Transplantação - IPST, 2016; Ministério da Saúde, 2015; Dunbar, 2020). No POP, salienta-se que os exames e condutas a serem tomadas serão da responsabilidade da equipe do setor. Deve-se: manter acesso venoso com solução fisiológica $0,9 \%$; verificar à beira de leito se o hemocomponente foi corretamente administrado no paciente destinado (identificação, tipagem sanguínea e fator Rh do paciente e da bolsa) para evidenciar possível erro de identificação; verificar sinais vitais; comunicar o ocorrido ao médico do paciente e à AT; notificar a reação ao serviço de hemoterapia por meio do aplicativo; enviar as amostras do receptor e da bolsa do HC para o serviço de hemoterapia e registrar em prontuário (Ministério da Saúde, 2015).

Os exames para diagnosticar a RT deverão ser solicitados pelo médico que intervir na reação transfusional imediata de acordo com a especificidade da suspeita diagnóstica (Center for Disease Control/Division of Healthcare Quality Promotion CDC/DHQP, 2018, 2020 \& National Healthcare Safety Network - NHSN, 2021). Essa informação deverá ser registrada imediatamente tanto no sistema de notificação do hospital pelo enfermeiro como o médico deverá preencher a Ficha de Notificações e Investigação de Incidentes Transfusionais (FIT) e também no prontuário a fim de que seja investigada conforme instrução da agência transfusional. Caso seja confirmada a RT, medidas preventivas serão implantadas e registradas no histórico transfusional. Reintegra-se que o óbito do paciente após uso de qualquer HC e que possa ter relação com a transfusão, deverá ser informado à ANVISA até 72h após o ocorrido (Agência Nacional de Vigilância Sanitária - ANVISA, 2015).

Não basta só suspeitar é preciso diagnosticar no período temporal para que medidas de mitigação sejam ativadas. Percebe-se que a Reação Febril Não Hemolítica (RFNH) e a Reação Alérgica (RALG) são prevalentes e facilmente reconhecidas e, indubitavelmente, são as recordistas em notificação, incluído a clínica pediátrica, em detrimento das reações cardiopulmonares que são subnotificadas pelo mundo (Grandi, Grell, Areco, \& Barbosa, 2018; Ghataliya et al., 2017; Roubinian, 2018). No entanto, as taxas de morbimortalidade das reações cardiopulmonares lideram entre os países 
desenvolvidos como EUA, Reino Unido, França (Center for Disease Control/Division of Healthcare Quality Promotion, 2018; Center for Disease Control/Division of Healthcare Quality Promotion \& NHSN - National Healthcare Safety Network, 2021; French National Agency for Medicine and Health Product Safety - ANSM, 2018; Serious Hazards of Transfusion - SHOT, 2019).

Em relação aos cuidados pós transfusionais foi elencado a conferência do registro de todo o processo no prontuário, monitorização por 24 horas e medidas de prevenção de recidivas de RT. No tocante a monitorização e ao registro, deve-se proceder as conjecturas do lapso temporal para as RT tanto para as reações imediatas quanto as tardias (Sahu \& Bajpai, 2020). Em relação aos sinais e sintomas, deve-se avaliar a alterações para prosseguir com a investigação (Center for Disease Control/Division of Healthcare Quality Promotion/National Healthcare Safety Network - CDC/DHQP/NHSN, 2021).

A literatura especializada na área abordada e descrita anteriormente, é composta, principalmente por manuais, protocolos e publicações científicas selecionados de instituições, sociedades e departamento governamentais e não governamentais, que embasam o trabalho de equipes interdisciplinares em diversos países. Esse conteúdo foi sintetizado para ilustrar o lapso temporal de cada RT e apontar os cuidados prévios e os cuidados posteriores a confirmação de RT. Esse levante bibliográfico foi disponibilizado para a equipe interdisciplinar, do hospital onde a pesquisa foi conduzida. Ainda foi elaborado e apresentado uma síntese levada em consideração a realidade de hospital público e as principais reações transfusionais descritas na literatura conforme a imputabilidade transfusional e as medidas de mitigação correspondentes (Quadro 3): 
Quadro 3 - Considerações do vínculo temporal e medidas preventivas para as Reações Transfusionais

\begin{tabular}{|c|c|c|}
\hline Transfusionais & $\begin{array}{l}\text { Lapso temporal e associação com sinais } \\
\text { e sintomas } \\
\text { (Imputabilidade) }\end{array}$ & Cuidados prévios à transfusão \\
\hline Reação Alérgica (RALG) & $\begin{array}{l}4 \text { horas após a cessação da transfusão e } \\
\text { sem outra evidência de correlação com os } \\
\text { riscos de reação alérgica a drogas, } \\
\text { alimentação e ambiente. Diagnóstico de } \\
\text { acordo com os sinais e sintomas. }\end{array}$ & $\begin{array}{l}\text { Sem comprovação científica para o uso de anti-histamínicos } \\
\text { previamente; Histórico de alergia medicamentosa e alimentar devem ser } \\
\text { avaliados; Utilização de } \mathrm{HC} \text { lavados (para } \mathrm{CH} \text { e plaquetas) após o } \\
\text { primeiro evento de reação anafilática ou na segunda vez em casos leves; } \\
\text { Identificar paciente portador de deficiência de IgA e utilizar HC } \\
\text { provenientes de pacientes com a mesma deficiência. }\end{array}$ \\
\hline Reação Hipotensiva (HIPOT) & $\begin{array}{l}15 \text { min após o início da transfusão } \\
\text { conforme critérios para a faixa etária e } \\
\text { responde rapidamente após a suspensão da } \\
\text { transfusão e tratamento. }\end{array}$ & $\begin{array}{l}\text { Avaliação prévia na fase do screening para uso de IECA previamente. } \\
\text { Suspensão prévia na fase pré-operatória. }\end{array}$ \\
\hline $\begin{array}{l}\text { Reação Febril não Hemolítica } \\
\text { (RFNH) }\end{array}$ & $\begin{array}{l}4 \text { horas e sem outras condições que } \\
\text { justifiquem os sinais e sintomas. }\end{array}$ & $\begin{array}{l}\text { Precisa da hemocultura do HC e do paciente para excluir Contaminação } \\
\text { Bacteriana (CB) e de dados clínicos para excluir RFH. } \\
\text { Sem comprovação científica para o uso de antipiréticos previamente. } \\
\text { Pode ser utilizado HC filtrados em casos de recorrência de RFNH. }\end{array}$ \\
\hline $\begin{array}{l}\text { Reação Febril Hemolítica } \\
\text { (RFH) }\end{array}$ & $\begin{array}{l}\text { Durante ou dentro das } 24 \text { horas da } \\
\text { cessação da transfusão. }\end{array}$ & $\begin{array}{l}\text { Identificação do paciente, da bolsa do } \mathrm{HC} \text { e da amostra para os testes de } \\
\text { compatibilidade com checagem dupla em todas as fases da transfusão; } \\
\text { reforçar teste de compatibilidade do grupo de } \mathrm{ABO} \text {, Rh e os demais. }\end{array}$ \\
\hline $\begin{array}{l}\text { Sobrecarga } \quad \text { Volêmica } \\
\text { Associada à Transfusão } \\
\text { (TACO) }\end{array}$ & $\begin{array}{l}\text { Realização de Raio x que comprove EAP } \\
\text { de origem cardiogênica na janela de } 12 \\
\text { horas. }\end{array}$ & $\begin{array}{l}\text { Avaliação do BH, PVC e DU comparando o antes, o durante e o após } \\
\text { uso de HC; Avaliação prévia do peso e da altura do receptor em relação } \\
\text { a quantidade e a dosagem ofertada; Uso de diurético com } 1 \mathrm{~h} \text { de } \\
\text { antecedência da transfusão para pacientes cardiopatas, pneumopatas e } \\
\text { renais (em pacientes não responsivos ao diurético, pode ser instituído } \\
\text { terapia renal substituta); administrar o HC uma unidade por vez } \\
\text { (aliquotar o HC); A administração do HC não deve ultrapassar uma } \\
\text { infusão } 2 \mathrm{ml} / \mathrm{min} \text { ou } 2 \mathrm{ml} / \mathrm{kg} / \mathrm{h} \text {. }\end{array}$ \\
\hline $\begin{array}{l}\text { Lesão Pulmonar Relacionada } \\
\text { à Transfusão (TRALI) }\end{array}$ & $\begin{array}{l}\text { Realização de Raio x que comprove EAP } \\
\text { de origem não cardiogênica na janela de } \\
06 \text { horas. }\end{array}$ & $\begin{array}{l}\text { Avaliação prévia de pacientes politransfundidos, doadoras multíparas e } \\
\text { pacientes críticos; Pesquisa de anti HLA no sangue do doador. Paciente } \\
\text { receber doação proveniente de nulíparas e do sexo masculino pode ser } \\
\text { ponderado; Manter a ventilação protetora em casos de pacientes em uso } \\
\text { de ventilação mecânica. }\end{array}$ \\
\hline $\begin{array}{l}\text { Dispnéia associada } \quad \text { à } \\
\text { Transfusão (TAD) }\end{array}$ & $\begin{array}{l}\text { Ocorre no período de } 24 \text { horas após a } \\
\text { interrupção da transfusão. }\end{array}$ & $\begin{array}{l}\text { Buscar exames comprobatórios de TRALI e TACO (radiografia de } \\
\text { tórax e hemogasometria) para definir critérios de exclusão. }\end{array}$ \\
\hline
\end{tabular}

Nota:1. Os sinais e sintomas e os sinais vitais precisam ser desconsiderados caso já apresentavam anteriormente à transfusão de acordo com os critérios de imputabilidade ou estejam fora do lapso temporal; 2. Para minimizar o risco para as reações como Distúrbio Metabólico e Hipotermia, deve ser elaborado o protocolo de Transfusão Maciça; 3. O Quadro 3 foi elaborado pelos autores que adaptaram o conteúdo referente a medidas de mitigação das Reações Transfusionais imediatas, de acordo com a literatura especializada produzida por organizações governamentais e não governamentais, além de pesquisadores especialistas na temática estudada.

Fonte: Elaborado pelos autores, adaptados das seguintes referências: CDC/NHSN, (2018-2020); SHOT, (2019), ISBT, (2018); CBS, (2017), Aubron et al., (2018); Vlaar et al., (2019); Kennedy et al., (2019); Goel, Tobian e Shaz, (2019); Ajmani, (2020); EBSERH, (2020), Bastos, (2015); ANVISA, (2015) e Silva et al., (2021).

\section{Considerações Finais}

O processo transfusional carece de bases consolidadas entre conjecturas: do aprendizado acerca dessa temática, do engajamento da equipe na monitorização e dos cuidados de todo o processo, além de qualificar as metas de segurança sobretudo a meta 1 (um) de identificação do paciente e a meta 2 (dois) de comunicação da equipe. Nessas fases, não são permitidos erros de comunicação nem de vigilância, porque as reações transfusionais podem ser procedentes de relação direta de aspectos assistenciais, além daqueles já inerentes ao doador e ao receptor.

Neste estudo foi apresentado a fundamentação teórica de forma organizada e sintética, extraído de um conteúdo denso e que embasou as etapas da construção de dois POP sobre Reações Transfusionais Imediata voltados para medidas de mitigação. Todo o conteúdo apresentado corrobora para a qualificação e validação do material produzido. Também se destaca a profundidade e problematização apresentadas no presente texto. Neste sentido, o artigo pode contribuir para as boas práticas transfusionais de hemocomponentes. 


\section{Referências}

Adkins, B. D., Lawicki, S., Johnson, M., \& Eichbaum, Q. (2019). Mild Allergic Transfusion Reactions. American Journal of Clinical Pathology, 151(3), 344348. https://doi.org/10.1093/ajcp/aqy150.

Agência Nacional de Vigilância Sanitária - ANVISA. (2015). Marco Conceitual e Operacional de Hemovigilância: Guia para a Hemovigilância no Brasil. Brasília.

Agência Nacional de Vigilância Sanitária - ANVISA. (2016). Hemovigilância no Brasil - Relatório consolidado 2007 - 2015. Brasilia. Retrieved from https://www.gov.br/anvisa/pt-br/assuntos/fiscalizacao-e-monitoramento/hemovigilancia/publicacoes/hemovigilancia-no-brasil-relatorio-consolidado-20072015.pdf/view.

Ajmani, P. S. (2020). Blood Test in Immunohematology and Blood Banking. In Immunohematology and Blood banking (pp. 77-101). Singapore: Springer Singapore. https://doi.org/10.1007/978-981-15-8435-0_7.

Alotaibi, Y. K., \& Federico, F. (2017). The impact of health information technology on patient safety. Saudi Medical Journal, 38(12), 1173-1180. https://doi.org/10.15537/smj.2017.12.20631.

Aubron, C., Aries, P., Le Niger, C., Sparrow, R. L., \& Ozier, Y. (2018). How clinicians can minimize transfusion-related adverse events? Transfusion Clinique et Biologique, 25(4), 257-261. https://doi.org/10.1016/j.tracli.2018.08.158.

Bastos, F. (2015). Guia para uso de hemocomponentes. In Ministério da Saúde. http://bvsms.saude.gov.br/bvs/publicacoes/guia_uso_he mocomponentes_2ed.pdf.

Bezerra, C. M., Cardoso, M. V. L. M. L., Silva, G. R. F. da, \& Rodrigues, E. da C. (2018). Creation and validation of a checklist for blood transfusion in children. Revista Brasileira de Enfermagem, 71(6), 3020-3026. https://doi.org/10.1590/0034-7167-2018-0098.

Bragas, L. Z. T. (2015). A importância da qualidade dos registros de enfermagem para gestão em saúde: estudo em hospital na região Noroeste do RS (Especialização em Gestão em Saúde). Universidade Federal do Rio Grande do Sul. Escola de Administração. Curso de especialização em Gestão em Saúde (UAB). https://lume.ufrgs.br/handle/10183/130291.

Cabedal, M. C. L. (2019). Hemoterapia e hemovigilância (Mestrado Integrado em Ciências Farmacêuticas). Universidade de Lisboa, Faculdade de Farmácia. Lisboa. https://repositorio.ul.pt/handle/10451/43329.

Canadian Blood Services - CBS. (2017). System Progress Reports. https://www.blood.ca/en/about-us/publications-and-reports/system-progress-reports.

Carneiro, V. S. M., Barp, M., \& Coelho, M. A. (2017). Hemotherapy and immediate transfusion reactions: action and knowledge of the nursing team. REME: Revista Mineira de Enfermagem, 21(0), 1031. https://doi.org/10.5935/1415-2762.20170041.

Castrillo, A., Arroyo, J. L., Romón, Í., \& Rivera, J. (2020). Compliance with temperature and time requirements during in-hospital distribution of blood components: A national survey among transfusion services. Transfusion and Apheresis Science, 59(6). https://doi.org/10.1016/J.TRANSCI.2020.102908.

Center for Disease Control/Division of Healthcare Quality Promotion. (2018). NHSN Biovigilance Component, Hemovigilance Module Protocol. The United States of America. Retrieved from https://www.cdc.gov/nhsn/acute-care-hospital/bio-hemo/index.html.

Center for Disease Control/Division of Healthcare Quality Promotion, \& NHSN - National Healthcare Safety Network. (2021). National Healthcare Safety Network biovigilance component hemovigilance module surveillance Protocol v2.6. The United States of America. https://www.cdc.gov/nhsn/pdfs/biovigilance/bv-hv-protocol-current.pdf.

Chasse, M., McIntyre, L., Tinmouth, A., Acker, J., English, S. W., Knoll, G., \& Fergusson, D. A. (2015). Clinical effects of blood donor characteristics in transfusion recipients: protocol of a framework to study the blood donor-recipient continuum. BMJ Open, 5(1), e007412-e007412. https://doi.org/10.1136/bmjopen-2014-007412.

Cherem, E. de O., Alves, V. H., Rodrigues, D. P., Pimenta, P. C. de O., Souza, F. D. L., \& Guerra, J. V. V. (2018). Processo de terapia transfusional em unidade de terapia intensiva neonatal: o conhecimento do enfermeiro. Texto \& Contexto - Enfermagem, 27(1), 1150016. https://doi.org/10.1590/010407072018001150016

Costa, C. O., Monteiro, I. B. S., Rodrigues, G. L. O., Monteles, A. O., \& Gomes, A. F. (2020). Atuação do enfermeiro na hemotransfusão: relato de experiência. Hematology, Transfusion and Cell Therapy, 42, 371-372. https://doi.org/10.1016/j.htct.2020.10.625.

Duarte, R. D., Silva, K. F. N. da, Félix, M. M. dos S., Tavares, J. L., Zuffi, F. B., \& Barbosa, M. H. (2017). Knowledge about blood transfusion in a critical unit of a teaching hospital. Bioscience Journal, 33(3), 788-798. https://doi.org/10.14393/BJ-v33n3-36196.

Empresa Brasileira de Serviços Hospitalares - EBSERH. (2021). Procedimento Operacional Padrão Código: POP ENF 15.1 Rotina de Instalação de Hemocomponentes para Transfusão. Rio de Janeiro.

Frantz, S. R. de S., Vargas, M. A. de O., DePires, D. E. P., Brito, M. J. M., Bitencourt, J. V. de O. V., \& Ribeiro, G. (2020). Nursing work and competence in hemotherapy services: an ergological approach. Revista Brasileira de Enfermagem, 73(3), e20180775. https://doi.org/10.1590/0034-7167-2018-0775.

French National Agency for Medicine and Health Product Safety - ANSM. (2018). Sécurité du Médicament et des produits de Santé. ANSM. L'ANSM publie le rapport d'activité hémovigilance 2018. https://ansm.sante.fr/qui-sommes-nous/publications-institutionnelles/publications-2019

Garraud, O., Sut, C., Haddad, A., Tariket, S., Aloui, C., Laradi, S., \& Andreu, G. (2018). Transfusion-associated hazards: A revisit of their presentation. Transfusion Clinique et Biologique, 25(2), 118-135. https://doi.org/10.1016/j.tracli.2018.03.002.

Gehrie, E. A., Hendrickson, J. E., \& Tormey, C. A. (2015). Measuring the influence of blood component infusion rate on recipient vital signs. Vox Sanguinis, 
109(4), 353-358. https://doi.org/10.1111/vox.12310.

Ghataliya, K., Kapadia, J., Desai, M., Mehariya, K., Rathod, G., Bhatnagar, N., \& Gajjar, M. (2017). Transfusion-related adverse reactions in pediatric and surgical patients at a Tertiary Care Teaching Hospital in India. Asian Journal of Transfusion Science, 11(2), 180. https://doi.org/10.4103/0973-6247.214348.

Gil, A. C. (2017). Como Elaborar Projetos de Pesquisa (6 ed.). Atlas.

Goel, R., Tobian, A. A. R., \& Shaz, B. H. (2019). Noninfectious transfusion-associated adverse events and their mitigation strategies. Blood, 133(17), 18311839. https://doi.org/10.1182/blood-2018-10-833988.

Gonçalves, M. I., Rocha, P. K., Anders, J. C., Kusahara, D. M., \& Tomazoni, A. (2016). Comunicação e segurança do paciente na passagem de plantão em unidades de cuidados intensivos neonatais. Texto \& Contexto - Enfermagem, 25(1), 2310014. https://doi.org/10.1590/0104-07072016002310014.

Gorski L, Hadaway L, Hagle ME, McGoldrick M, Orr M, D. D. (2016). Infusion therapy standards of practice. Infusion Nurses Society, 39(18). Retrieved from www.journalofinfusionnursing.com.

Grandi, J. L., Grell, M. C., Areco, K. C. N., \& Barbosa, D. A. (2018). Hemovigilância: a experiência da notificação de reações transfusionais em Hospital Universitário. Revista Da Escola de Enfermagem Da USP, 52, e03331. https://doi.org/10.1590/s1980-220x2017010603331.

Guerado, E., Medina, A., Mata, M. I., Galvan, J. M., \& Bertrand, M. L. (2016). Protocols for massive blood transfusion: when and why, and potential complications. European Journal of Trauma and Emergency Surgery: Official Publication of the European Trauma Society, 42(3), 283-295. https://doi.org/10.1007/s00068-015-0612-y.

Gurgel, A. P., DeMelo, V. S., Leitão, J. S., Studart, R. M. B., Bonfim, I. M., \& Barbosa, I. V. (2019). Paciente crítico: segurança em terapia transfusional mediante lista de verificações. Revista Brasileira de Ciências Da Saúde, 23(4), 525-534. https://doi.org/10.22478/ufpb.2317-6032.2019v23n4.37205.

Harewood, J., Ramsey, A., \& Master, S. R. (2021). Hemolytic Transfusion Reaction. In StatPearls. StatPearls Publishing. https://www.ncbi.nlm.nih.gov/books/NBK448158/.

Holland, J., Peralta, R. M., Moss, R. L., Feane, K., \& Uprichard, J. (2020). A single-centre review of iatrogenic anaemia in adult intensive care. Transfusion Medicine, 30(3), 196-200. https://doi.org/10.1111/tme.12674.

Hospitais Universitários Federais - EBSERH. (2020). Manual de Condutas em Reações Transfusionais (Vol. 1). Pernambuco. http://www2.ebserh.gov.br/documents/210672/5180417/MANUAL+002+SAT+CONDUTAS+NA+REACAO+TRANSFUSIONAL+versao+1+\%281\%29.pdf /d89673dc-c985-496a-8f06-705d205ebb75.

Hulse, W., Bahr, T. M., Fredrickson, L., Canfield, C. M., Friddle, K., Pysher, T. J., \& Christensen, R. D. (2020). Warming blood products for transfusion to neonates: In vitro assessments. Transfusion, 60(9), trf.16007. https://doi.org/10.1111/trf.16007.

Instituto Português do Sangue e da Transplantação - IPST. (2016). Algoritmos de notificação o de Reação es Adversas em Receptores Sistema Português de Hemovigilância. http://www.hemovigilancia.net/docs/Algoritmos.pdf.

International Society of Blood Transfusion/International Haemovigilance Network - ISBT/IHN. (2019). Definição revisada de caso de vigilância internacional de sobrecarga circulatória associada à transfusão: uma classificação estudo de validação de contrato. https://www.isbtweb.org/.

Kennedy, M. S., Harmeníng, D., \& Justín Rhees. (2019). Transfusion Therapy. In Modern Blood Banking and Transfusion Practices (pp. 355-373). Elsevier.

Knopfelmacher, A. M. (2020). Transfusion Reactions. In Oncologic Critical Care (pp. 1177-1190). Cham: Springer International Publishing. https://doi.org/10.1007/978-3-319-74588-6_107.

Kolankiewicz, A. C. B., Schmidt, C. R., Carvalho, R. E. F. L. de, Spies, J., Dal Pai, S., \& Lorenzini, E. (2020). Patient safety culture from the perspective of all the workers of a general hospital. Revista Gaúcha de Enfermagem, 41, e20190177. https://doi.org/10.1590/1983-1447.2020.20190177.

Lim, Y. A., Kim, J., \& Park, C. (2020). Early recognition of possible transfusion reactions using an electronic automatic notification system for changes in vital signs in patients undergoing blood transfusions. Transfusion, 60(9), 1950-1959. https://doi.org/10.1111/TRF.15931/FULL.

Lima, A. A. de, Silva, G. P. da, Rocha, S. M. da, \& Barbosa, E. L. (2016). A importância do enfermeiro durante a reação transfusional aguda: revisão da literatura. Revista Recien - Revista Científica de Enfermagem, 6(17), 45. https://doi.org/10.24276/rrecien2358-3088.2016.6.17.45-56.

López Lozano, B. (2015). Análisis del conocimiento enfermero ante una transfusión sanguínea: revisión bibliográfica. Revista Científica de Enfermería, O(10). https://doi.org/10.14198/recien.2015.10.04.

Malvik, N., Leon, J., Schlueter, A. J., Wu, C., \& Knudson, C. M. (2020). ABO-incompatible platelets are associated with increased transfusion reaction rates. Transfusion, 60(2), 285-293. https://doi.org/10.1111/trf.15655.

Marconi, M. de A., \& Lakatos, E. M. (2021). Metodologia do Trabalho Científico (9 ed.). Atlas.

Medecins Sans Frontieres - MSF. (2019). Transfusion: Guide pratique à l'usage des médecins, infirmiers et personnel de santé gérant des activités de transfusion sanguine Do (Édition 20). Medecins Sans Frontieres - MSF.

Ministério da Saúde. Portaria n 1.820, de 13 de agosto de 2009 Dispõe sobre os direitos e deveres dos usuários da saúde. (2009). Diário Oficial da União.

Ministério da Saúde. Portaria n. 158, de 4 de fevereiro de 2016. Redefine o regulamento técnico de procedimentos hemoterápicos. (2016). Diário Oficial da Únião.

Moncharmont, P. (2018). Platelet component transfusion and alloimmunization: Where do we stand? Transfusion Clinique et Biologique, 25(3), 172-178. https://doi.org/10.1016/j.tracli.2018.01.003. 
Mora, A., Ayala, L., Bielza, R., Ataúlfo González, F., \& Villegas, A. (2019). Improving safety in blood transfusion using failure mode and effect analysis. Transfusion, 59(2), 516-523. https://doi.org/10.1111/trf.15137.

Najafpour, Z., Hasoumi, M., Behzadi, F., Mohamadi, E., Jafary, M., \& Saeedi, M. (2017). Preventing blood transfusion failures: FMEA, an effective assessment method. BMC Health Services Research, 17(1), 453. https://doi.org/10.1186/s12913-017-2380-3.

Negi, G., Gaur, D. S., \& Kaur, R. (2015). Blood transfusion safety: A study of adverse reactions at the blood bank of a tertiary care center. Advanced Biomedical Research, 4(2), 237. https://doi.org/10.4103/2277-9175.168604.

New Zealand Blood Service. (2016). Transfusion Medicine Handbook: Third Edition. 1-171.

Dunbar, N. M. (2020). Does ABO and RhD matching matter for platelet transfusion? Hematology, 2020(1), 512-517. https://doi.org/10.1182/hematology.2020000135.

Oliveira, J. L. C., Cervilheri, A. H., Haddad, M. do C. L., Magalhães, A. M. M., Ribeiro, M. R. R., \& Matsuda, L. M. (2020). Interface entre acreditação e segurança do paciente: perspectivas da equipe de enfermagem. Revista da Escola de Enfermagem da USP, 54. https://doi.org/10.1590/s1980$220 \times 2018053703604$.

Organização Mundial de Saúde - OMS. (2017). Recomendações - OMS. Retrieved July 18, 2021, from https://www.bloodless.com.br/pt/recomendacoes-daorganizacao-mundial-de-saude-oms/.

Panch, S. R., Montemayor-Garcia, C., \& Klein, H. G. (2019). Hemolytic Transfusion Reactions. New England Journal of Medicine, $381(2), 150-162$. https://doi.org/10.1056/NEJMra1802338.

Patidar, G. K., \& Kaur, D. (2018). Audit and education: Role in safe transfusion practice. Asian Journal of Transfusion Science, 12(2), 141-145. https://doi.org/10.4103/ajts.AJTS_135_17.

Polares, A. C., Costa, B. L. O., Souto, F. de L., Queiroz, I. P., Barbosa, B. dos S., \& Queiroz, I. P. (2020). Ato transfusional: Ocorrência de não-conformidades no processo de hemotransfusão em pacientes imunossuprimidos. Brazilian Journal of Health Review, 3(5), 11542-11555. https://doi.org/10.34119/bjhrv3n5015 .

Raval, J. S., Griggs, J. R., \& Fleg, A. (2020). Blood Product Transfusion in Adults: Indications, Adverse Reactions, and Modifications. American Family Physician, 102(1), 30-38. Retrieved from https://pubmed.ncbi.nlm.nih.gov/32603068/.

Reis, V. N., Paixão, I. B., Perrone, A. C. A. de S. J., Monteiro, M. I., \& Santos, K. B. (2016). Transfusion monitoring: care practice analysis in a public teaching hospital. Einstein (São Paulo), 14(1), 41-46. https://doi.org/10.1590/S1679-45082016AO3555.

Ribeiro, W. A., Faillace, G. B. D., Fassarella, B. P. A., Neves, K. do C., Fassarella, M. B., Silva, A. S. A. C. S. da, \& Farias, B. S. (2020). Protagonização do médico na segurança do paciente nas reações transfusionais: uma revisão integrativa. Research, Society and Development, $9(7)$, e572974597. https://doi.org/10.33448/rsd-v9i7.4597.

Rodrigues, A. D., \& Ribeiro, L. R. (2021). Sistemas sanguíneos, incompatibilidade e procedimentos alternativos à transfusão. Brazilian Journal of Development, 7(2), 13007-13027. https://doi.org/10.34117/bjdv7n2-085.

Roubinian, N. (2018). TACO and TRALI: biology, risk factors, and prevention strategies. Hematology, $2018(1), \quad 585-594$. https://doi.org/10.1182/asheducation-2018.1.585.

Sahu, A., \& Bajpai, M. (2020). Determining the true incidence of acute transfusion reactions: Active surveillance at a specialized liver center. Hematology, Transfusion and Cell Therapy, 42(4), 326-332. https://doi.org/10.1016/j.htct.2019.09.006.

Saito-Benz, M., Sandle, M. E., Jackson, P. B., \& Berry, M. J. (2019). Blood transfusion for anaemia of prematurity: Current practice in Australia and New Zealand. Journal of Paediatrics and Child Health, 55(4), 433-440. https://doi.org/10.1111/jpc.14222.

Santos, A. A. B. da S., Teixeira, J. D. C., Pereira, L. D. C. da S., \& Silva, A. L. de A. (2020). Conhecimento de enfermeiros sobre reações transfusionais: revisão integrativa. Revista Recien - Revista Científica de Enfermagem, 10(31), 65-73. https://doi.org/10.24276/rerecien2020.10.31.65-73.

Santos, L. X., Santana, C. C. de A. P., \& Oliveira, A. de S. B. (2021). Hemotransfusion under the perspective of nursing care. Revista Online de Pesquisa Cuidado é Fundamental, 13, 65-71. https://doi.org/10.9789/2175-5361.rpcfo.v13.7458.

Schaffhausser, C. J., Faria, J. C. P., Suano-Souza, F. I., \& Sarni, R. O. S. (2020). Red blood cell prescription and recognition of transfusion reactions by pediatricians. Einstein (São Paulo), 18. https://doi.org/10.31744/einstein_journal/2020AO5446.

Semple, J. W., Rebetz, J., \& Kapur, R. (2019). Transfusion-associated circulatory overload and transfusion-related acute lung injury. Blood, 133(17), 18401853. https://doi.org/10.1182/BLOOD-2018-10-860809.

Serious Hazards of Transfusion - SHOT. (2019). New or Unclassifiable Complications of Transfusion (UCT). Retrieved from https://www.shotuk.org/wpcontent/uploads/myimages/19.-New-or-Unclassifiable-Complications-of-Transfusion-UCT.pdf.

Shah Zeb, \& Tazeen Saeed Ali. (2020). Factors associated with the compliance of standard precaution; review article. Journal of the Pakistan Medical Association, 71(2B), 1-14. https://doi.org/10.47391/JPMA.416.

Silva, A. B., DeOliveira, A. A., Silva, J. de O. M., Santos, G. K. de B. B., Esteves, R. B., \& DaSilva, Â. M. (2021). Caracterização epidemiológica das reações transfusionais imediatas em um hospital público de ensino. Research, Society and Development, 10(9), e5110916635. https://doi.org/10.33448/rsdv10i9.16635.

Simon, T. L., McCullough, J., Snyder, E. L. (Edward L., Solheim, B. G., \& Strauss, R. G. (2016). Rossi's principles of transfusion medicine (5th ed.; Wiley- 
Research, Society and Development, v. 10, n. 11, e32101119257, 2021

(CC BY 4.0) | ISSN 2525-3409 | DOI: http://dx.doi.org/10.33448/rsd-v10i11.19257

Blackwell, Ed.).

Spilka, J., \& Goobie, S. M. (2020). Manejo Transfusional Perioperatório no Paciente Pediátrico. Boston: Word Federation of Societies of Anaesthesiologists. Retrieved from https://resources.wfsahq.org/wp-content/uploads/418_ATOTW_PORTUGUES.pdf.

Sullivan, K. D., Vu, T., Richardson, G., Castillo, E., \& Martinez, F. (2015). Evaluating the Frequency of Vital Sign Monitoring During Blood Transfusion: An Evidence-Based Practice Initiative. Clinical Journal of Oncology Nursing, 19(5), 516-520. https://doi.org/10.1188/15.CJON.516-520.

Tang, C.-H., Huang, Y.-X., Lin, Y.-X., \& Yuan, M. (2020). [Analysis of Related Factors of Adverse Transfusion Reactions]. Zhongguo Shi Yan Xue Ye Xue Za Zhi, 28(3), 972-976. https://doi.org/10.19746/j.cnki.issn.1009-2137.2020.03.042.

Tavares, J. L., Barichello, E., DeMattia, A. L., \& Barbosa, M. H. (2015). Factors associated with knowledge of the nursing staff at a teaching hospital on blood transfusion. Revista Latino-Americana de Enfermagem, 23(4), 595-602. https://doi.org/10.1590/0104-1169.0024.2593.

Vargas Bermúdez, Z. M., \& Vargas Bermúdez, Z. M. (2019). Guía de cuidados de enfermería para la administración de la sangre y sus componentes (Revisión integrativa). Enfermería Actual de Costa Rica, (37), 168-187. https://doi.org/http://dx.doi.org/10.15517/revenf.v0ino.37.36531.

Veiga, V. C., \& Rojas, S. S. O. (2018). Como reduzir os riscos de eventos adversos em transfusão. In Associação de Medicina Intensiva Brasileira (1st ed., pp. 59-75). Porto Alegre: Artmed Panamericana.

Vieira, C. M. de A. S., \& Santos, K. B. (2020). The knowledge of the nursing team on transfusion of hemocomponents: an integrated review. Revista de Pesquisa Cuidado é Fundamental Online, 12, 517-524. https://doi.org/10.9789/2175-5361.rpcfo.v12.8623.

Vlaar, A. P. J., Toy, P., Fung, M., Looney, M. R., Juffermans, N. P., Bux, J., \& Kleinman, S. (2019). A consensus redefinition of transfusion-related acute lung injury. Transfusion, 59(7), 2465-2476. https://doi.org/10.1111/trf.15311.

Whittaker, R., McRobbie, H., Bullen, C., Rodgers, A., Gu, Y., \& Dobson, R. (2019). Mobile phone text messaging and app-based interventions for smoking cessation. Cochrane Database of Systematic Reviews, 2019(10). https://doi.org/10.1002/14651858.CD006611.pub5.

Witosz, K., Wojnarowicz, O., \& Krzych, Ł. J. (2021). Iatrogenic blood loss due to daily laboratory testing and the risk of subsequent anaemia in intensive care unit patients: case series. Acta Biochimica Polonica, 68(1), 135-138. https://doi.org/10.18388/abp.2020_5525.

Yoon, U., Abdullah, M., Elia, E., \& Herman, J. (2018). Intraoperative Diagnosis and Management of Acute Hypotensive Blood Transfusion Reaction (AHTR): A Report of Two Cases. American Journal of Case Reports, 19, 1283-1287. https://doi.org/10.12659/AJCR.910642. 